\title{
Bias Mitigation in Data Sets
}

\author{
Shea Brown \\ ForHumanity, BABL \\ Ryan Carrier \\ ForHumanity \\ Merve Hickok \\ sheabrown@bablai.com ryan@forhumanity.center \\ ForHumanity, AIethicist.org \\ Adam Leon Smith \\ ForHumanity, Dragonfly \\ merve@lighthousecareerconsulting.com adam@wearedragonfly.co
}

\begin{abstract}
Tackling sample bias, Non-Response Bias, Cognitive Bias, and disparate impact associated with Protected Categories in three parts/papers, data, algorithm construction, and output impact. This paper covers the Data section.

Index Terms-artificial intelligence, machine learning, bias, data quality
\end{abstract}

\section{INTRODUCTION}

Bias is a loaded word. It has serious implications and gets used a lot in the debates about algorithms that occur today. Research around bias in AI/ML systems often draws in fairness, even though they are two very different concepts. To avoid confusion and help with bias mitigation, we will keep the concepts separate. In its widest sense, bias can originate, or be found, across the whole spectrum of decisions made about processing data. Bias can originate in human-societalinstitutional interactions that are codified in data [1] [2] [3] [4], in labels [5] [6], in methods, and in trained models Consequently, these sources of bias can lead to unfairness in the outcomes resulting from the algorithm. It can even be found in how we choose to interpret those algorithmic outcomes [7] [8]. Therefore, with a word like bias, that is broadly and sometimes casually used, this paper chooses to address bias discretely. We are breaking the big word "bias" down as much as we can into building blocks so we may expose the crucial elements for which humanity should demand mitigation. This allows us to frame the concept more specifically, from the ground up, with uncomplicated discernible solutions and techniques to create a system for managing bias in its many forms.

In Figure 1 below, the highlighted yellow boxes are the areas of bias we are discussing and mitigating in this paper. Subsequent papers will tackle other areas of bias which are addressed in Independent Audit of AI Systems bias mitigation procedures.

There are several points in the life cycle of algorithmic design where we need to mitigate bias. The three types of bias we aim to mitigate can occur in each part of the life cycle: Design, Development, Deployment, and Operation. These life cycle stages are shown in Figure 2.

Sample Bias, Non-Response Bias and Cognitive Bias introduce and increase the likelihood of a variety of risks that manifest when we do not manage our datasets well. A few examples are listed below:

1) Legal risk - non-compliance with regulation, for example anti-discrimination [9] or data privacy regulation [10].
2) Ethical and reputational risk - perpetuation of stereotypes and discrimination, failure to abide by a Code of Ethics or social responsibility policy

3) Functional risk - bad validity, poor fit, and misalignment with the intended scope, nature, context and purpose of the system

The desire of the authors, and ForHumanity's Independent Audit of AI Systems, is to explain and document mitigations related to all bias in its many manifestations in the data input phase of AI/ML model development.

Circling back to the societal concern with the statistical notion of bias - societies, under the law of different jurisdictions, have determined that bias is prohibited with respect to certain data characteristics - human characteristics, such as race, age, gender etc. Therefore, while bias can occur in any statistic, of any kind, legal mitigation of bias need only occur because stakeholders desire it, or in satisfaction of the law. Therefore, ForHumanity introduces the following definitions:

- Protected Category Variables - Defined by jurisdiction legally, may include race, age, gender, religion, ability/disability, sexual orientation, color, nation of origin, socioeconomic class, etc.

All of our audit criteria, specifically our bias mitigation sections, rely upon the legal definition of these Protected Category Variables from each jurisdiction. Said differently, our criteria are designed to remediate bias that is found associated with these variables (and Proxy Variables or derived Inferences). In the context of algorithmic decisions, we need to ensure that these variables are analyzed at the data level so that decisions do not become obscured behind veils.

- Proxy Variables - Features of a dataset that correlate strongly with a Protected Category Variable that, when combined with Inferences, can be used to associate a Data Subject with a Protected Category Variable

- Inferences - assumption or conclusion reached by a data processing algorithm, which may not be treated as fact and shall be labelled as such

To illustrate, part of ForHumanity's audit criteria are listed below in Table I.

These four audit criteria explain that trainingtesting/validation data or processing data is allowed to use any of these types of data which can point back to Protected Category Variables (PCV). ForHumanity requires bias mitigation when PCVs or Proxy Variables/Inferences are 


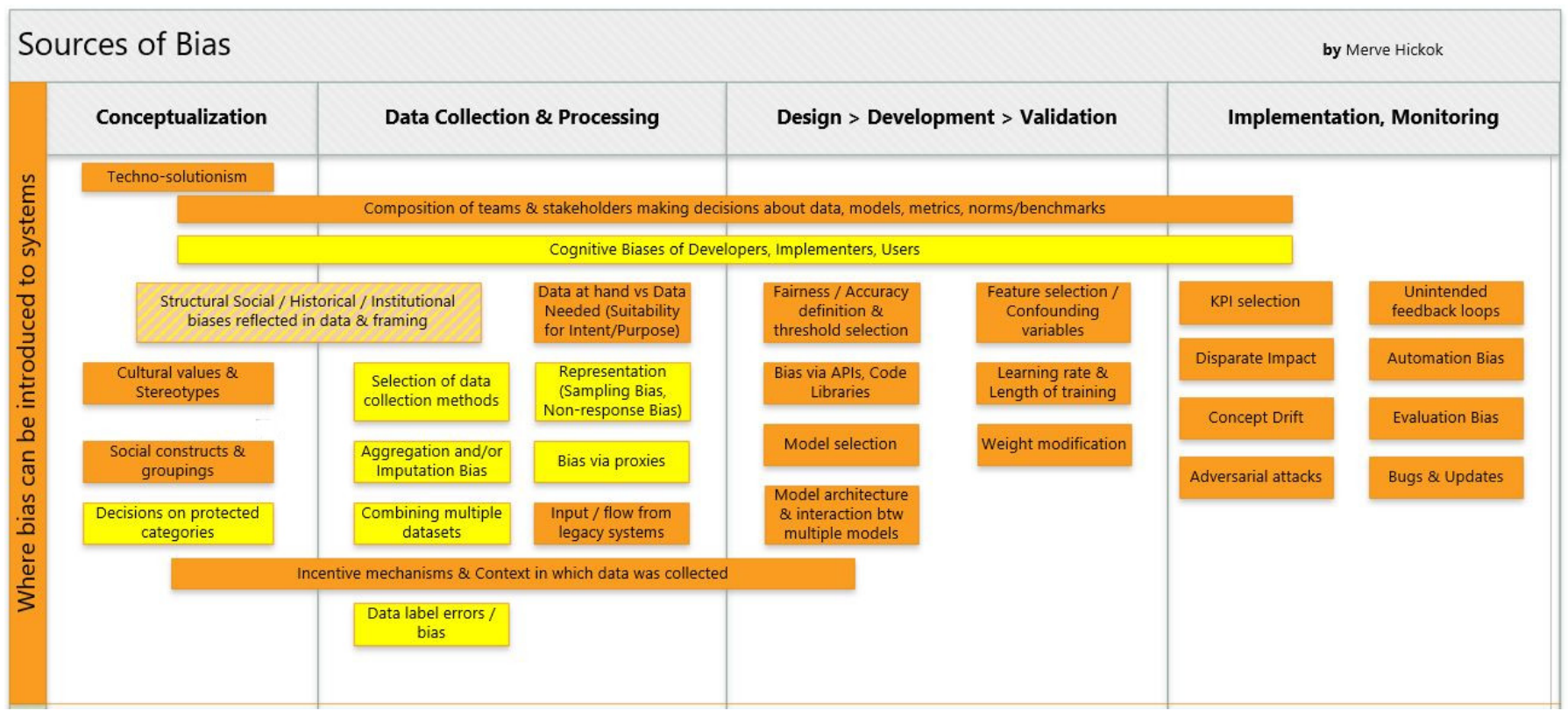

Fig. 1. Sources of Bias

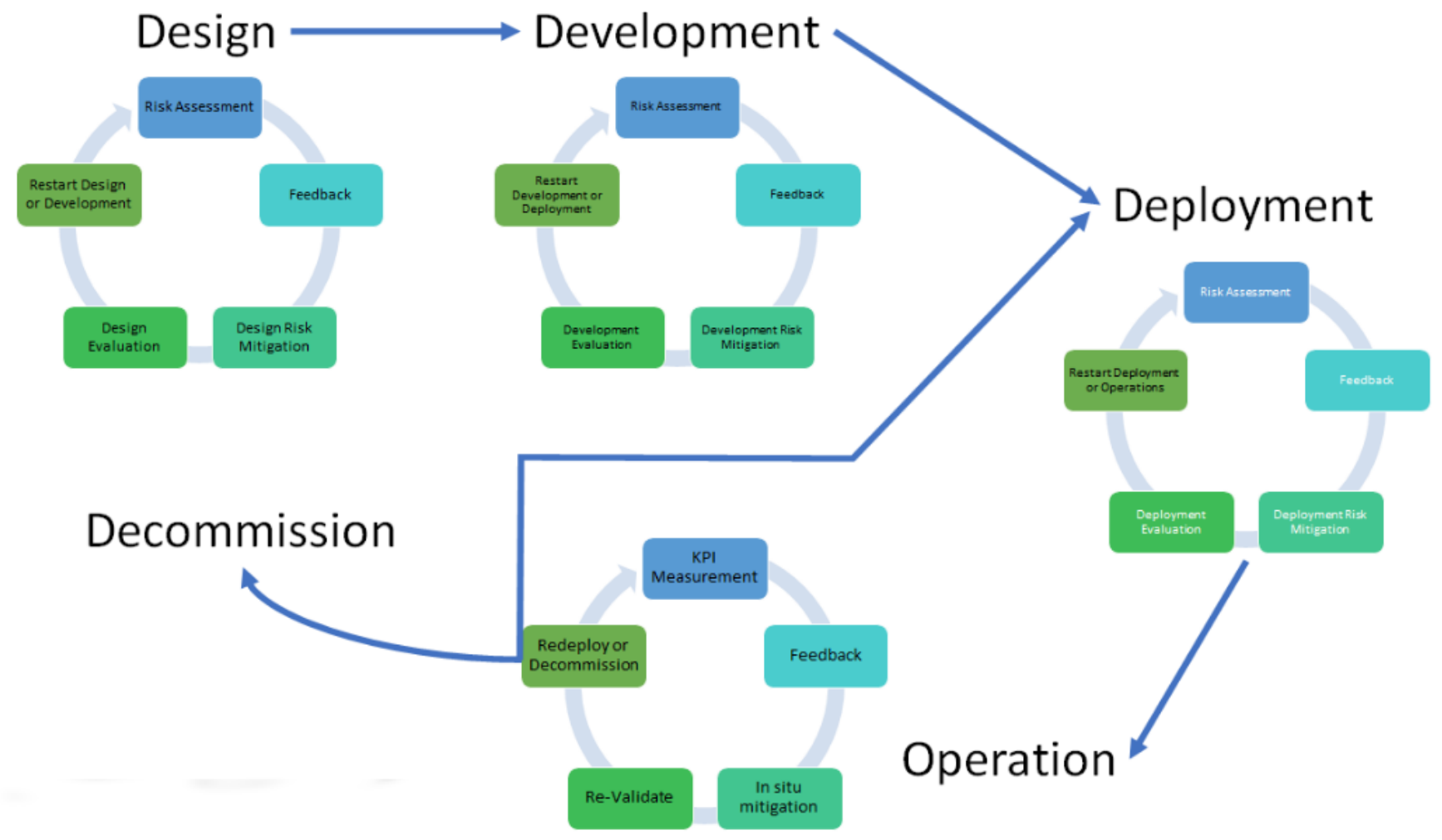

Fig. 2. ForHumanity AI/ML life cycle 
TABLE I

EXAMPLE AUDIT CRITERIA

\begin{tabular}{|c|c|}
\hline Audit question & Audit backup \\
\hline $\begin{array}{l}\text { The entity's training-testing/validation } \\
\text { data may have Protected Category } \\
\text { Variables. }\end{array}$ & $\begin{array}{l}\text { Internal logs, registers } \\
\text { or databases }\end{array}$ \\
\hline $\begin{array}{l}\text { The entity's training-testing/validation } \\
\text { and processing data may use Inferences. } \\
\text { The entity's processing data may have } \\
\text { Protected Category Variables. }\end{array}$ & $\begin{array}{l}\text { Internal logs, registers } \\
\text { or databases } \\
\text { Internal logs, registers } \\
\text { or databases }\end{array}$ \\
\hline $\begin{array}{l}\text { The Ethics Committee shall determine } \\
\text { the number of discrete categories for } \\
\text { each Protected Category Variable and } \\
\text { shall offer an "other or no response" } \\
\text { category. }\end{array}$ & $\begin{array}{l}\text { Internal procedure } \\
\text { manual }\end{array}$ \\
\hline
\end{tabular}

present in any training or processing dataset. Other algorithms may go through bias mitigation, but it is not required.

\section{BUILding Block 1 - Exploratory Data ANALYsis}

Sample bias in training-testing/validation data is a commonly cited cause of bias in implemented machine learning (ML) systems, and so as an early part of developing such systems a review process, that we are calling Input Data Testing, is required to determine risk factors. Part of that testing is exploratory data analysis (EDA) [11]. This work can be conducted as soon as training-testing/validation data is available, and the risk factors can later be tested using the implemented system. This analysis must be conducted on the final, aggregated dataset. The training-testing/validation data should be analyzed, and the following questions examined:

1) Is there a Protected Category Variable (PCV) used as a feature?

2) Is there another feature (Proxy Variable) that correlates with a PCV ?

3) Is there less than a representative amount of data associated with a PCV ?

4) Are the training and testing/validation datasets collected using different methodologies?

5) Are the Statistical Characteristics different between the training data and the testing/validation data?

6) Are the labels unreliable or inconsistent?

If the answer to any of the questions is affirmative, then there is a risk of sample bias causing bias in system outcomes. In order to answer the first two questions it is necessary to obtain the training-testing/validation dataset, to review the features, and to calculate the correlations between features. In Figure 3, below, you can see that AGE has a strong positive correlation with an arbitrary feature, NOX. This means that even if AGE is not included in the selected features (as it is likely a PCV), then it is possible that NOX will act as a Proxy Variable.

The third question is harder, it can be broken down into several further questions:

- What are the appropriate and possible values or ranges for each PCV, and how can they be appropriately grouped? For example, referring back to the correlation matrix above, does the strong correlation with NOX hold true across all ages? Or are there some age groups where this correlation is very different? In the example of ethnicity, are the values selected reflective of societal, and technical considerations?

- Is the number of records associated with each PCV group statistically significant? Each group will have a certain number of records available in the trainingtesting/validation data. How statistically significant the training-testing/validation data depends on (a) the number of features; (b) the size of the target population; (c) the degree of variation in the feature values; (d) the correlation between each feature and the target variable.

- Are the features present consistently for each PCV group? ML models don't handle null values. It is tempting, when cleaning data, to either drop the whole record, or to guess (impute) the missing data. Consequently, if features are missing disproportionately for one PCV group, there is a risk of introducing sample bias during the development process. Alternatively, any imputation logic might exhibit other kinds of bias, like Cognitive Bias or stereotypes.

- For supervised learning, is the target variable (whatever our ML model is trying to predict) available with a similar frequency for each PCV group? In supervised learning, training is conducted with labeled data, that is, data with the target variable attached. Firstly, that label might not be available for all records, and records that do not have the label will not be used for training-testing/validation. That means, whilst data may be present it might be dropped or changed later in the model development process. Secondly, it may be that the distribution of label values associated with a particular PCV in the training data, does not match reality 1 . Thirdly, the labels attached to the data might not be correct.

The fourth and fifth questions are aimed at ensuring that the metrics used to test the performance of the final model are accurate and representative of its true performance. This requires that the testing/validation datasets are statistically similar to the training data, without being identical. The importance of the method used to collect the data might be very important here, for example, the time of day that it is collected, the people or records that are sampled, the people involved in the collection, and any Cognitive Bias that they have, and the technical techniques used. All of these choices about the method can impact the statistical distribution of the data, including representativeness.

From question five, a 'statistical characteristic' is any measurable parameter of the data sample. This could be, e.g., the mean value or standard deviation of one of the features. In the ISO 3534-4:2014 [12] standard, this would be called an 'estimate' of a 'population parameter', where the trainingtesting/validation data are 'samples' from a larger 'population'. Questions 4 and 5 are essentially asking; are the training data and testing/validation data similar samples from the same population?

\footnotetext{
${ }^{1}$ Even if the data matched the reality $100 \%$, the reality is, almost in all cases, not an equal or equitable world
} 


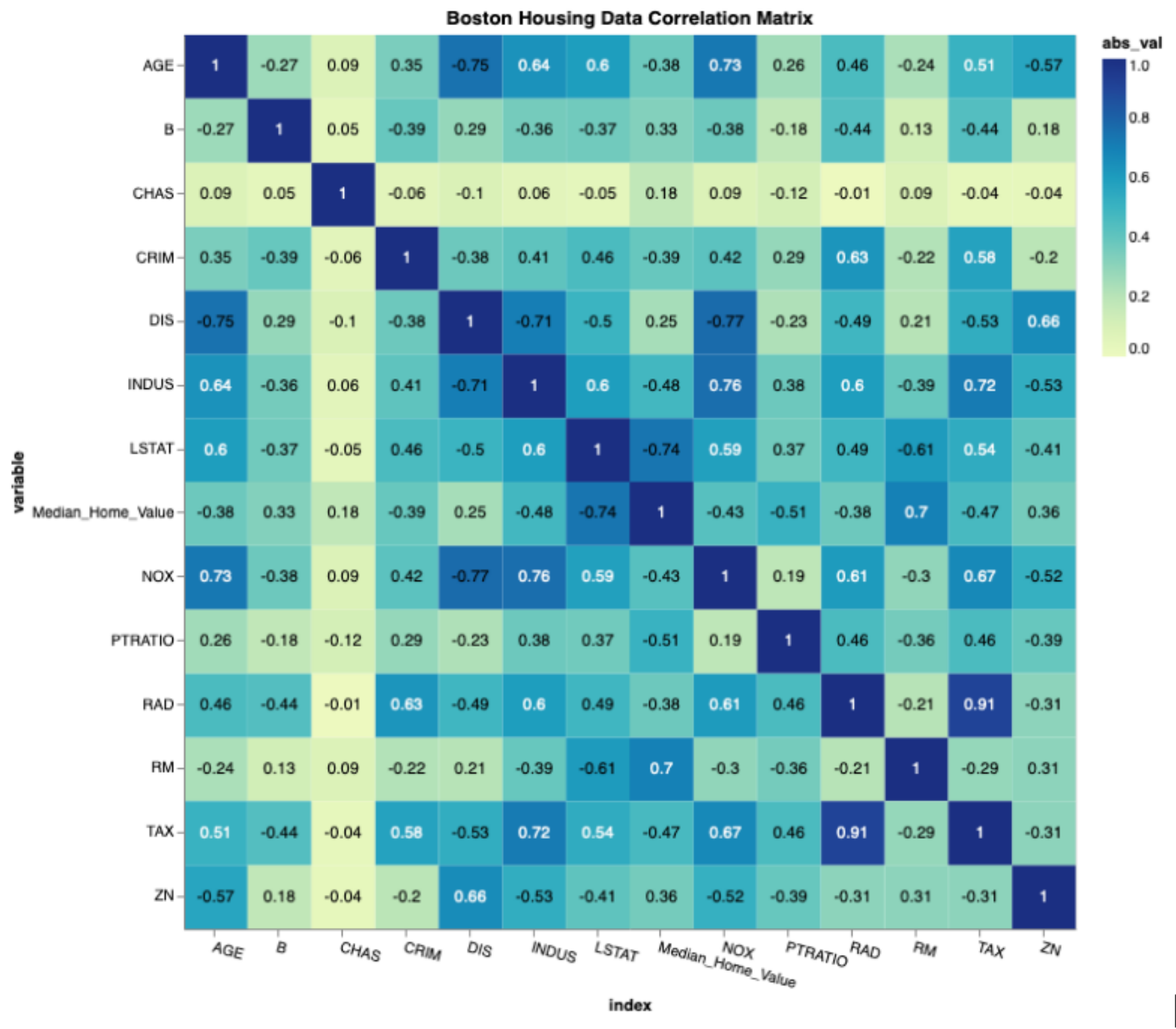

Fig. 3. A correlation matrix

It is necessary to use tools to conduct this analysis, to calculate correlations, profile data, and visualize it. The most obvious being Python's open-source frameworks, however various commercial tools also exist for this task [See Appendix for some open-source and commercially available tools]. As illustrated by the complex nature of the questions above, it is important that use of any tool does not become a check-box exercise, and is instead as one analysis tool within a toolbox and a rigorous process.

Unless you are using unsupervised machine learning, raw data in your data set needs labeling. The quality of your data is crucial to the success of your model, and great attention is particularly needed for labeling. In fact, a consistently biased application of labels is worse for the model performance than a randomly incorrect labeling. There might be several reasons for your data to be inaccurately labeled. Sometimes the labels are wrong in defining the item, or not applied consistently, and sometimes there might be multiple labels that could be applied to an item. In addition to sample bias, we now need to consider the other types of bias mentioned at the beginning of the paper. These are Cognitive Biases, those biases that are introduced by the human brain. There are hundreds of known Cognitive Biases [13]. ForHumanity seeks reasonable consideration by the Algorithmic Risk Committee, to examine and consider whether these biases pose risks. Explanatory Note 1 of Independent Audit of AI Systems User Guide v1.1 [14] provides a list of 15 different measures that are considered reasonable efforts to remediate Cognitive Biases. These must be examined, analyzed, considered and documented when deployed for mitigation of Cognitive Biases. 
One simple example of this bias is where labels are crowdsourced, that is, outsourced to humans. In the absence of training and clear guidelines, it is easy for the personal biases and experiences of the labelers to be reflected in how they annotate data.

Another example is Non-Response Bias, that is the degree to which certain groups of people might not participate in some survey, or interactive labelling process, to the same degree as others. This might refer to the physical, or technological restrictions limiting the inclusion of certain PCV groups (the elderly, the poor, etc) and measures must be taken to remedy the Non-Response Bias, or explain and disclose the Non-Response Bias. A biased label can cause a model to ignore the observation when making a prediction / classification; or to count towards a wrong observation. So whether you are crowdsourcing the labeling (annotation, tagging), or using automation to assign values, a number of safeguards need to be in place to ensure a better quality:

- Just as the diversity and representativeness of your dataset and the stakeholders involved in the life cycle of AI systems matter, diversity of the labelers also matters. The experiences and background of labelers can impact how they perceive what they see and are labeling; or the annotation might be impacted by their preferences. This diversity can be used to understand different ways of annotating data and foresee possible deviations.

- In addition to selecting diverse and reliable labelers, developers/project managers also need to ensure that the labelers are trained according to clear and precise labeling guidelines.

- Labeled benchmark data sets can be developed. The guidelines and this benchmark set can be used as a standard to compare labeler consistency and accuracy.

- The same data could be labeled/annotated by multiple labelers, which could then be reviewed, or averaged.

- A random audit of labeled data can be conducted for quality checks.

At the end of the exercise, a list of risks should exist, and some degree of prioritization can be applied by an ethics committee, officer, or Algorithmic Risk Committee. These risks may, or may not manifest as biases in the actual outcomes of the system, because the model developers could:

- Apply synthetic data to minority classes to rebalance the dataset [15]

- Remove real data to rebalance the dataset

- Apply hyper-parameters ${ }^{2}$ that minimise the bias

- Remove problematic features

- Limit the scope or context of use of the system

- Remediate incorrect labels

Each of the risks should have a documented mitigation in the development process, and later, a documented set of test

\footnotetext{
${ }^{2}$ These are any values that the developer can choose to modify the training process. These could be weights that are applied to the data based on class (e.g., down-weight the majority data) or some other manipulation that might off-set the imbalance.
}

results from model testing and other test processes that show that the development mitigations were successful.

\section{A. Mapping Sample Bias to IAAIS audit criteria and definitions}

To begin the mitigation process we have to further dissect our algorithmic processes and build our foundation of terminology. Around the world, different jurisdictions agree certain classes of people with shared traits are disadvantaged historically and determine that members of these classes with common characteristics should be protected against discrimination Protected classes (or categories) are defended with laws to codify fair and equal treatment. Since these biases are often driven from society itself, each society has wrestled with the groups that it needs to protect and thus there is no global uniformity on which groups should be protected. Thus, ForHumanity is forced to adapt our system to each jurisdiction's definition of Protected Category Variables. We use the term Relevant Legal Frameworks to refer back to the law and categorizations based upon the appropriate jurisdictions for the system being considered.

Terms in bold black are the IAAIS (Independent Audit of AI Systems) terminology.

There are a few kinds of data we want to consider and define clearly:

1) Realized - observed values of Protected Category Variables used to determine representativeness in the data (in IAAIS = Statistical Characteristics )

2) Expected - a hypothetical benchmark(s) established for an algorithmic process or expected by the law and society. In the ForHumanity process, these are established by the ethics committee or an ethics officer to determine reasonable representativeness of Protected Category Variables (in IAAIS = Baseline Population Benchmarks)

1) Realized data can further be subdivided into

2) Training data - used to test, design, and tweak the model for production

3) Testing/Validation data - provides an independent evaluation of a model fit on the target dataset while tuning the model's hyperparameters

4) Processing data - data observed once the model is in production

Training-testing/validation, and Processing data have Statistical Characteristics and Baseline Population Benchmarks. Statistical Characteristics and Baseline Population Benchmarks should have similar distributions as trainingtesting/validation data sets and processing data sets. Significant deviations in Statistical Characteristics or Baseline Population Benchmarks across the training-testing/validation and processing data sets create a risk of sample bias and/or representativeness issues between the data sets.

Below, in Table II, are additional related audit criteria that require the Algorithmic Risk Committee to ensure that training-testing/validation data sets are sufficiently similar. 
TABLE II

EXAMPLE AUDIT CRITERIA

\begin{tabular}{l|ll} 
Audit question & \multicolumn{2}{|l}{ Audit backup } \\
\hline $\begin{array}{l}\text { The Algorithmic Risk Committee shall } \\
\text { compare, examine and analyze training } \\
\text { data and validation/testing data set for } \\
\text { statistical deviations. }\end{array}$ & manual procedure \\
$\begin{array}{l}\text { The Algorithmic Risk Committee shall } \\
\text { determine that training data and valida- }\end{array}$ & $\begin{array}{l}\text { Internal logs, registers } \\
\text { or databases }\end{array}$ \\
$\begin{array}{l}\text { tion/testing data sets that are statistically } \\
\text { similar. }\end{array}$ & \\
$\begin{array}{l}\text { The Algorithmic Risk Committee shall } \\
\text { explain and disclose material deviations }\end{array}$ & Internal procedure \\
between the training data and valida- \\
tion/testing data
\end{tabular}

\section{B. A note on "reasonable"}

The reader might wonder about the term "reasonable". Please recognize that this is a carefully chosen term with legitimate legal meaning. "Reasonable" in a legal context means the following [16]:

The most obvious example of vagueness is the word reasonable. It introduces an objective standard in the contract. The term reasonable places a limit on discretionary power or the effect of overly strict obligations. Where it limits the exercise of discretionary power, it requires that a party is able to explain its performance (or failure to perform as expected).

Where the term reasonable is included with the aim of reducing the 'harshness' of strict contract clause, it introduces a common sense approach to the interpretation of what may normally be expected from a party's performance.

The standard of 'reasonableness' is one that is usually determined by reference to a well-informed third party with the same expertise acting under the same circumstances.

See also Independent Audit of AI Systems USER GUIDE $\mathrm{v} 1.1$.

\section{Building BLOCK 2 - Establishing BENCHMARKS (Baseline Population Benchmarks)}

This problem is critical for examining sample bias. Let us illustrate with an example [17] [18] . A firm wants to hire an entry-level computer programmer and has decided that the skill requires a bachelor's degree in computer science. When we consider if our data set underrepresents, overrepresents or reasonably represents gender (only considering male/female and not the spectrum of gender identity for this example 3 , what Baseline Population Benchmark - what expected mean should we compare our data against?

Here are a few reasonable benchmarks (Baseline Population Benchmarks):

1) Global gender diversity $(51.5 \%$ women $v 48.5$

\footnotetext{
${ }^{3}$ This is also an example of bias. When questions like this are asked where only restricted choices are offered not reflective of reality of options, some individuals may be excluded because they are forced to a category they might not identify with but do not have an alternative. The cumulative effect of such practices is data gets more biased in representation and identities and voices of some groups are consistently cancelled.
}

2) Male/Female bachelor's of computer science degree holders ( $82 \%$ men v $18 \%$ women)

3) Or some other measure related to a local workforce, like McAllen Tx, where women outpace men by $28.5 \%$, the highest in America

4)

This should highlight a few key issues:

1) Any of those benchmarks are at least reasonable (in the legal sense of the term)

2) Benchmarks may themselves have a bias, especially where bias already exists in society, and the data set available. Benchmarks can capture systemic bias which will impact outcomes if unmitigated

3) Whatever you choose, bias will exist versus the other reasonable benchmarks and likely still against your benchmark of choice

4) Considering multiple benchmarks may help to measure and manage when bias mitigation techniques may be helpful while avoiding an overcorrection.

5) Choice is involved in settling on the benchmarks and that choice is one that involves an ethical decision.

Focusing on ethical decision-making, we hope the reader sees that selecting the benchmark(s) involves an application of principles and rules, the details of which are beyond the scope of this paper. For clarity sake, ForHumanity defines Ethical as follows:

Ethical - In the context of automated and intelligent systems, (ethical) is a set of principles and rules concerning moral obligations and regards for the rights of humans and nature, which may be specified by a given profession or group

Ethical Choice - awareness of a set of options to be made using a set of principles and rules concerning moral obligations and regards for the rights of humans and for nature, which may be specified by a given profession or group. The result, outcome or judgment is made using a shared moral framework or set of moral principles based upon the entity's Code of Ethics

Therefore, the first step in establishing benchmarks is to avoid the inherent conflict-of-interest for designers and developers and let experts representing diverse perspectives trained in Ethical Choice establish the benchmarks for the algorithmic process: Failure to involve an ethics committee sets up designers and developers for failure as they are apt to best-fit their choice to the data rather than to make the Ethical Choice(s) of benchmarks to avoid the extra step of bias mitigation altogether. ForHumanity handles this in our audit criteria as follows in Table III.

TABLE III

EXAMPLE AUDIT CRITERIA

\begin{tabular}{l|l} 
Audit question & Audit backup \\
\hline The ethics committee shall be responsi- & Internal procedure \\
ble for determining the Baseline Popu- & manual \\
lation Benchmarks for all algorithmic & \\
processes that have Protected Category & \\
Variables. &
\end{tabular}


This approach ensures that instances of Ethical Choice are given proper scrutiny, and facilitates technical input from designers and developers as well as ethical input. The ForHumanity risk assessment process requires diverse inputs and multistakeholder feedback to provide input into both design and development portions of the life cycle and support risk assessment feedback loops. The Ethics Committee, trained in benchmark-setting, can respond to these risks and feedback loops to provide reasonable benchmarks for the process. The design and development process should not conclude without these risk mitigation steps being accomplished. However, these risk mitigations cannot be achieved solely by examining data.

This is where other notion of bias, in the form of fairness, algorithmic harms and discrimination usually comes into picture. Notably, in US, as an example, Civil Rights Act of 1964 prohibits discrimination as a result of race, color, religion, sex, or national origin. Equal Employment Opportunity Commission (EEOC) prohibits it within the context of employment, or Fair Housing Act within the context of housing; or, for example, New York City code adds more categories within the boundaries of NYC, or Charter of Fundamental Rights of EU (Title 3) regulates it across EU member states.

Below, we establish some criteria for the way an ethics committee and/or a trained ethics officer can make effective benchmarking (Baseline Population Benchmarks) decisions.

\section{Building Block 3 - Has the Ethics Committee DETERMINED THAT THE DATASET IS REASONABLY REPRESENTATIVE?}

The recent EU Commission proposed Artificial Intelligence Act [19] Article 10.3 calls for overall Data Quality which IAAIS assigns to the Algorithmic Risk Committee for comprehensive examination and analysis. Defined in IAAIS as follows: for both processing data and training data, is the data up-todate, of high quality, applicable to the algorithm, complete and representative. Article 10.3 also specifically references data "should be representative". As an undefined and "soft law" concept, ForHumanity considers "representativeness" as a question of Ethical Choice which the Ethics Committee should be responsible to determine. Questions of Ethical Choice are also the domain of a Code of Data Ethics, which is a policy that Independent Audit of AI Systems requires each entity to produce. A Code of Data Ethics should establish the moral framework that the company chooses to abide by with regard to representativeness. IAAIS imposes a few ethical expectations upon all entities which are explicit through the whole body of work, such as governance, accountability, oversight, disclosure, and transparency. Beyond these tenets, the entity and its ethics committee must identify its moral framework and apply it rigorously.

With that foundation, we offer a set of questions and examinations we "suggest" an ethics officer or Ethics Committee use to discuss the technical aspects with designers and developers. The goal is for the Ethics Committee to assert that to a "reasonable" standard that the data set is representative. The questions are as follows and they are not intended to be comprehensive, but rather a representative sample to establish an understanding of the nature of the data set and provide the Algorithm Risk Committee the confidence to proceed with the algorithm to additional phases of compliance. Documentation of these deliberations, conclusions, and mitigations are necessary to satisfy the "reasonableness" standard.

TABLE IV

EXAMPLE AUDIT CRITERIA

\begin{tabular}{l|l} 
Audit question & Audit backup \\
\hline The ethics committee shall be responsi- & Internal procedure \\
ble to examine and analyze the data set, & manual \\
to determine that it is reasonably repre- & \\
sentative for the intended scope, nature, \\
context and purpose of the algorithm, \\
including Protected Category Variable \\
and features.
\end{tabular}

The following questions are suggested for an Ethics Committee to discuss representativeness:

1) Can or should the PCV be eliminated from the dataset or are they a valid feature of the dataset?

2) For each Protected Category Variable has the committee decided upon the discrete breakdowns for consideration (e.g. race or ethnic group - how many subcategories are meaningful and representative given the context of the system)?

3) Is there any PCV excluded that might be meaningful to the algorithmic architecture?

4) For each PCV does the committee feel that members are accurately represented in the data set?

a) Is an equality benchmark (where all PCVs made equal) used?

b) Is a combination of benchmarks used?

c) Is a public benchmark used?

d) How were the benchmarks determined and have the reasons and benchmarks been disclosed?

5) For each discrete PCV, is the combination of features and Proxy Variables representative of that Protected Category?

6) For the intersection of PCVs that are appropriate given the context of the system, for example, gender and race, are the features representative of those Protected Categories?

7) How has incomplete data been processed?

8) Was synthetic or imputed data used? What was the method for creating the synthetic data and was it used to manage representativeness?

9) Did the Ethics Committee create or design any intentional bias mitigation-related data collection techniques?

10) How were those techniques measured and what was the threshold for determining that they were needed?

11) What is the threshold for data "age"? How old can data be and still be meaningful and relevant to the algorithm?

12) Has the Ethics Committee compared the training data to the testing/validation data and determined both statistical and representativeness similarities and meaningful differences to justify a reasonable testing/validation set? 
13) Are there any PCV categories excluded, if so why? And has the committee justified the exclusions with transparency and disclosure?

14) Are there any PCV exclusions based on statistical thresholds or other quantitative measures?

15) Are there features present in the data set which might lead to PCV exclusions, especially if intended in the algorithmic architecture?

16) Was there a data label audit/labeler training in place?

When this work is completed we reach our final step at least with respect to the data. The most valuable tools for accountability, governance and oversight: 1) transparency and 2) disclosure 3) Explainability

\section{Building Block 4 - Data Disclosure Document} (DDD)

Bias mitigation to ForHumanity can be accomplished in many ways, however, there is always one final stalwart set of solutions which are accomplished together in our Data Disclosure Document (DDD), which is also a publicly disclosed document:

1) Disclosure

2) Transparency

3) Explainability

Once we have taken all practical steps to mitigate bias (building blocks 1-3), as discussed in the beginning, we know we will not achieve perfection - there are no completely unbiased algorithms The DDD provides a description of the mitigation steps taken in the context of data.

The Data Disclosure Document is a statistical and informative presentation of data from both training-testing/validation and processing data provided publicly. It is designed to be transparent and explain the steps an entity has taken to identify and remediate bias at the data input level. It requires that considerations, choices, and justifications made in data decisions be explicit. We have provided a tabular list of Data Disclosure Document items:

1) All Baseline Population Benchmarks for all Protected Category Variables that are present in the dataset have been identified and explained

2) The origin of the data, and the collection, processing, and labeling procedures.

3) All training-testing/validation data broken out for each Protected Category Variable's Statistical Characteristics remediate bias at the data input level. It requires that considerations, choices, and justifications made in data decisions be explicit. We have provided a tabular list of Data Disclosure Document items:

a) Realized Mean (\% of the total dataset)

b) Realized Count

c) Output/documentation/commentary from Building Block 3 - Data Representativeness

4) All Processing data are broken out for each Protected Category Variable's Statistical Characteristics (a representative snapshot averaged over a reasonable time interval consistent with the frequency of the change in data - taken for reporting purposes)

a) Realized Mean (\% of the total dataset tracked over time)

b) Realized Count

c) Frequency of evaluation

d) Output/documentation/commentary from Building Block 3 - Data Representativeness

5) Mitigation steps for Cognitive Bias and Non-Response Bias are explained and disclosed.

It is hoped and anticipated that the DDD will provide researchers and advocates the transparency that is required to raise issues, to learn, to examine, and to understand the models upon which they rely. This process uses our key risk management techniques:

1) Eliminate conflicts of interest

2) Increase accountability, governance, and oversight

3) Seek objective problem solvers (often ethics officers)

4) Mitigate bias to a "reasonable" standard

5) Finish with transparency, disclosure, and explainability in the DDD

Accountability is further enhanced when the process requires and fosters greater transparency. Now, not only is someone watching, and checking, but the whole world is able to see the work, to review and critique crucial elements of disclosure and compliance. Transparency and disclosure create a feedback loop. Once information is disclosed, critics can provide constructive critique into the process and facilitate future improvements. Hence transparency itself is not an end goal but a means to improve. Accountability combined with transparency creates a virtuous circle of improvement and development.

\section{CONCLUSION}

ForHumanity attempts to take a comprehensive approach to mitigate bias in datasets and algorithms. Beyond looking at the Statistical Characteristics of bias, and questions of representativeness, we require that the Algorithmic Risk Committee consider the following sources of bias:

1) Data Entry Point Attacks (dedicated audit criteria)

2) Concept Drift (KPIs and frequency of testing audit requirements to track)

3) Bias in Architectural inputs, choices and design

4) Outcomes, adverse impact, publicly disclosures

These points will be covered in a follow-up paper in order to complete coverage and explanation of bias mitigation designed and implemented in Independent Audit of AI Systems.

Listed below in Table $\mathrm{V}$ are the audit rules used to examine each element of bias mitigation covered in this paper. 
TABLE V

BIAS MITIGATION AUDIT CRITERIA

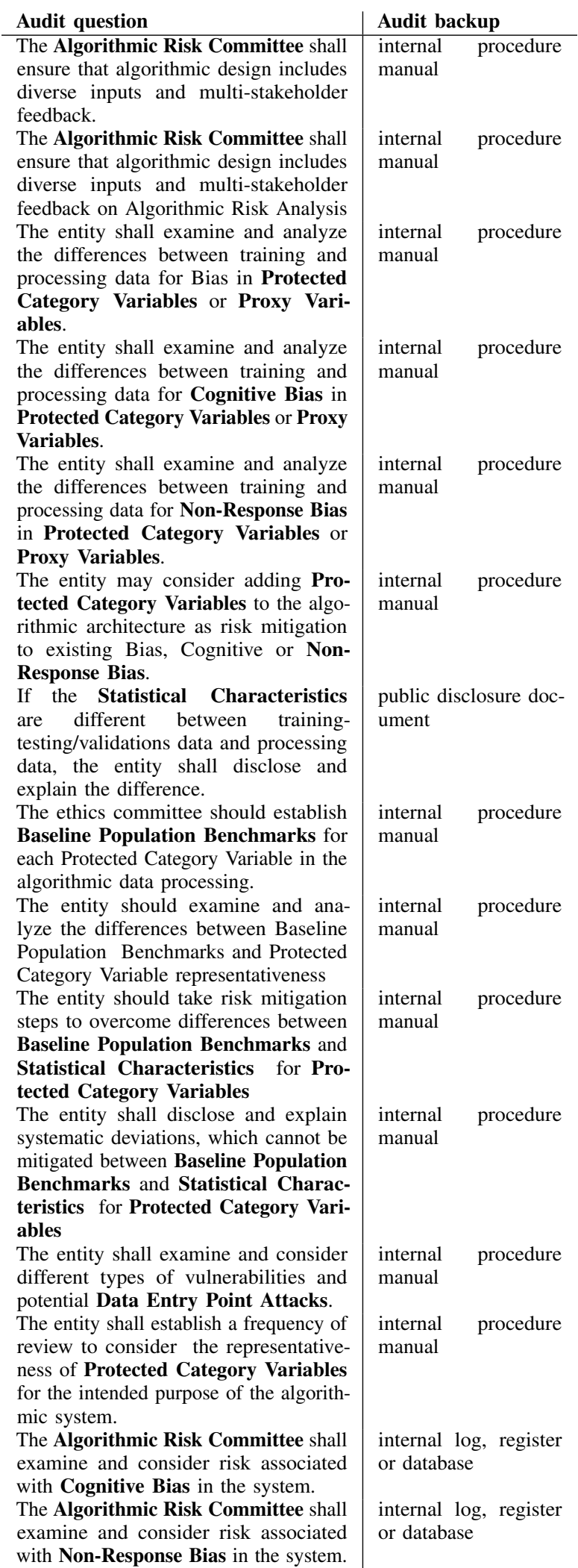

In Table VI, below, we further consider the frequency of data changes and model adaptation from learning-based algorithms:
TABLE VI

MONITORING AUDIT CRITERIA

\begin{tabular}{l|l} 
Audit question & Audit backup \\
\hline The DPO/DCC shall document Key & Internal procedure \\
Performance Indicators (KPIs) for & manual \\
each system to determine continued & \\
efficacy in the context of the data pro- & \\
cessing. & \\
The DPO/DCC shall document the fre- & Internal log, register \\
quency of assessing Key Performance & or database \\
Indicators (KPIs) in conjunction with & \\
the frequency of change in the system. & \\
An Ethics officer on the Algorithmic & Internal procedure \\
Risk Committee shall examine, and & manual \\
consider instances of Ethical Choice & \\
in the establishment of KPI's by the & \\
CDO/DPO. &
\end{tabular}

Each of these items deserves a paper to examine implementation and compliance measurement. This paper has focused on bias mitigation procedures for datasets. Mitigation is a carefully chosen word highlighting the certainty that bias can never be completely eliminated. This simple fact reminds us that bias, in its many forms, is something we must be ever vigilant about in all of our AIs and Autonomous systems. Or duty...ForHumanity is to ensure the best possible results for all humans and especially for those who need our care the most. These steps are a start, but there remains much work to be done.

\section{ApPendix - SAmple of Open ACcess And Closed ACCESS TOOLS FOR BiAs Mitigation}

The list below does not constitute an endorsement by ForHumanity. It is a non-exhaustive list added for reference purposes. If utilized, special attention should be paid to each tool's disclosed purpose, structure, shortcomings. They should be used not as a checklist activity, but one of the many tools available in a toolbox).

- ACLU, Algorithmic Equity Toolkit https://www.aclu-wa. org/sites/default/files/media-documents/aekit_digital.pdf

- AISP, A Toolkit for Centering Racial Equity Throughout Data Integration https://www.aisp.upenn.edu/wp-content/ uploads/2020/07/AISP-Toolkit_5.27.20.pdf

- Fairly AI, Validation Engine https://www.fairly.ai/

- Google, Playing with AI Fairness: What-if Tool https: //pair-code.github.io/what-if-tool/ai-fairness.html

- IBM, AI Fairness 360 Open Source Toolkit https://aif360. mybluemix.net//

- LinkedIn Fairness Toolkit (LiFT) https://github.com/ linkedin/LiFT

- IBM, AI Explainability 360 Toolkit https://aif360. mybluemix.net/

- IBM, AI FactSheets 360 Toolkit https://aifs360.mybluemix. net//

- IBM, Adversarial Robustness Toolbox https: //adversarial-robustness-toolbox.readthedocs.io/en/latest/

- Linux Foundation, AI Explainability 360 https://lfai. foundation/projects/aiexplainability360/ 
- TensorFlow, Fairness Indicators https:/github.com/ tensorflow/fairness-indicators

- TensorFlow, Model Analyzer https://www.tensorflow.org/ tfx/model_analysis/get_started

- TensorFlow, Responsible AI - Model Remediation https: //www.tensorflow.org/responsible_ai/model_remediation

- FairLean https://fairlearn.org/

- Spatial Equity Data Tool https://github.com/UrbanInstitute/ ui-equity-tool

- Error Analysis https://erroranalysis.ai/

- Interpret ML https://interpret.ml/

- ELI5 https://eli5.readthedocs.io/en/latest/overview.html

- LIME https://github.com/marcotcr/lime

- SHAP https://github.com/slundberg/shap

\section{REFERENCES}

[1] R. Richardson, J. M. Schultz, and K. Crawford, "DIRTY DATA, BAD PREDICTIONS: HOW CIVIL RIGHTS VIOLATIONS IMPACT POLICE DATA, PREDICTIVE POLICING SYSTEMS, AND JUSTICE," NEW YORK UNIVERSITY LAW REVIEW, vol. 94, p. 42.

[2] Algorithmic bias detection and mitigation: Best practices and policies to reduce consumer harms.' urlhttps://www.brookings.edu/research/algorithmic-bias-detectionand-mitigation-best-practices-and-policies-to-reduce-consumer-harms/ (accessed Jun. 11, 2021).

[3] L. Sweeney, "Discrimination in Online Ad Delivery," p. 36.

[4] D. K. Citron and F. Pasquale, "THE SCORED SOCIETY: DUE PROCESS FOR AUTOMATED PREDICTIONS," WASHINGTON LAW REVIEW, vol. 89, p. 34.

[5] D. Tsipras, S. Santurkar, L. Engstrom, A. Ilyas, and A. Madry, "From ImageNet to Image Classification: Contextualizing Progress on Benchmarks," arXiv:2005.11295 [cs, stat], May 2020, Accessed: Jun. 11, 2021. [Online]. Available: urlhttp://arxiv.org/abs/2005.11295

[6] J. Zhang, V. S. Sheng, Q. Li, J. Wu, and X. Wu, "Consensus algorithms for biased labeling in crowdsourcing," Information Sciences, vol. 382-383, pp. 254-273, 2017, doi: urlhttps://doi.org/10.1016/j.ins.2016.12.026

[7] K. Goddard, A. Roudsari, and J. C. Wyatt, "Automation bias: a systematic review of frequency, effect mediators, and mitigators," J Am Med Inform Assoc, vol. 19, no. 1, pp. 121-127, Jan. 2012, doi: 10.1136/amiajnl-2011000089.

[8] "Task 5: Prepare implementers to deploy your AI system," Sep. 18, 2020.urlhttps://ico.org.uk/for-organisations/guide-to-data-protection/keydata-protection-themes/explaining-decisions-made-with-artificialintelligence/part-2-explaining-ai-in-practice/task-5-prepare/ (accessed Jun. 11, 2021).

[9] "Protections Against Discrimination and Other Prohibited Practices," Federal Trade Commission, Jul. 16, 2013. urlhttps://www.ftc.gov/siteinformation/no-fear-act/protections-against-discrimination (accessed Jun. 11, 2021).

[10] European Parliament. REGULATION (EU) 2016/679 OF THE EUROPEAN PARLIAMENT AND OF THE COUNCIL on the protection of natural persons with regard to the processing of personal data and on the free movement of such data, and repealing Directive 95/46/EC (General Data Protection Regulation), 2016.

[11] urlhttps://www.jstor.org/stable/2237638

[12] "ISO 3534-4:2014(en), Statistics — Vocabulary and symbols — Part 4: Survey sampling."

[13] "List of Cognitive Biases", Wikipedia. Jun. 09, 2021 Accessed: Jun. 11, 2021. [Online]. Available: urlhttps://en.wikipedia.org/w/index.php?title=List_of_cognitive biases

[14] Independent Audit of AI Systems User Guide v1.1, ForHumanity, 2021

[15] M. Randall, "Five Ethical Risks to Consider before Filling Missing Race and Ethnicity Data", p. 14. Accessed: Jun. 11, 2021. [Online]. Available: urlhttps://www.urban.org/sites/default/files/publication/103830/fiveethical-risks-to-consider-before-filling-missing-race-and-ethnicity-dataworkshop-findings_0.pdf
[16] "Reasonable in contracts - meaning of vague terms," Weagree urlhttps://weagree.com/drafting-principles/1-general-draftingprinciples/1-4-vagueness-and-ambiguity/a-avoid-vague-terms/i-vagueterms-reasonable/ (accessed Jun. 11, 2021).

[17] "Women in Computer Science - ComputerScience.org," Get an Education the World Needs - ComputerScience.org, Oct. 15, 2020. urlhttps://www.computerscience.org/resources/women-in-computerscience/ (accessed Jun. 11, 2021).

[18] "United States Gender Ratios." urlhttps://www.states101.com/genderratios/global/unitedstates (accessed Jun. 11, 2021).

[19] "Proposal for a Regulation laying down harmonised rules on artificial intelligence - Shaping Europe's digital future." urlhttps://digitalstrategy.ec.europa.eu/en/library/proposal-regulation-laying-downharmonised-rules-artificial-intelligence (accessed Jun. 18, 2021). 